\title{
Computational model of the heating system
}

\author{
R. Barauskas*, V. Grigaliunas**, M. Gudauskis**, L. Obcarskas**, K. Sarkauskas***, \\ A. Vilkauskas**, A. Zvironas** \\ *Department of Applied Informatics, Faculty of Informatics, Kaunas University of Technology, Studentu St. 50, LT-51368 \\ Kaunas, Lithuania, E-mail: rimantas.barauskas@ktu.lt \\ **Institute of Mechatronics, Kaunas University of Technology, Studentu st. 56, LT-51424 Kaunas, Lithuania, \\ E-mail: mechatronics@ktu.lt \\ ***Department of Automation, Faculty of Electrical and Electronics Engineering, Kaunas University of Technology, \\ Studentu st. 48, LT-51367 Kaunas, Lithuania, E-mail: kasark@ktu.lt
}

cross $^{\text {ref }}$ http://dx.doi.org/10.5755/j01.mech.22.1.12244

\section{Introduction}

A lot of today's publications are allocated for optimization, automatization and application of artificial intelligence in heating systems creation. This is done for economic [1,2] and ecological [3,4] reasons. New types of heating systems are analyzed, designed and developed. Some publications present solar energy [3], heat pump with air to air, air to water, geothermal [5], district-heating systems [6], etc.

The primary objective of smart heating systems is to maintain a comfortable temperature in the rooms. The heating system is controlled by thermostats with defined time parameters. In $[1,2]$ a computer controlled system for increase of efficiency of the heating system is proposed. It follows human presence in the rooms and analyzes their activity. Dependent on the human activity, the system controls the heating system parameters values.

The aim of our paper is to propose a method for increasing electric efficiency of older houses heating systems by making simple changes in heating pumps. It has been demonstrated that appropriate start-stop control of the pump may result in significant reduction of the overall pump operation time.

The heating system under investigation consists of the boiler, heater and connecting pipes (Fig. 1, a). The heater is situated in a closed box, which imitates the room to be heated. In the future the air volume within the box is referred to as the heated space (HS).

The boiler is assumed to be an ideal one, the temperatures of which are always constant and equal $\left(60^{\circ} \mathrm{C}\right)$ at the zones of connection of the supply and return pipes. In this model the temperature of the HS is assumed to be uniform throughout its volume. The temperature of the HS is influenced by the heat exchange processes through its outer surface, as well as, through the surface of the heater in contact with HS. The heat conductivity and surface convection coefficients of the heater are assessed by assuming it as an equivalent pipe. The transient heat transfer problem is solved under assumption that the heating fluid flow rate is a known time function. The aim of the analysis is to find the time law of the fluid flow rate, which ensures minimum power consumption by the pump. Steady temperature of HS within given tolerances must be maintained.

The model is developed by providing mathematical formulations of:

- diffusive heat exchange (conduction) in the fluid, in the walls of the pipes and of the heater;

- convective heat transfer due to the fluid flow;

- convective heat transfer through between the fluid (air) and adjacent solid structure.

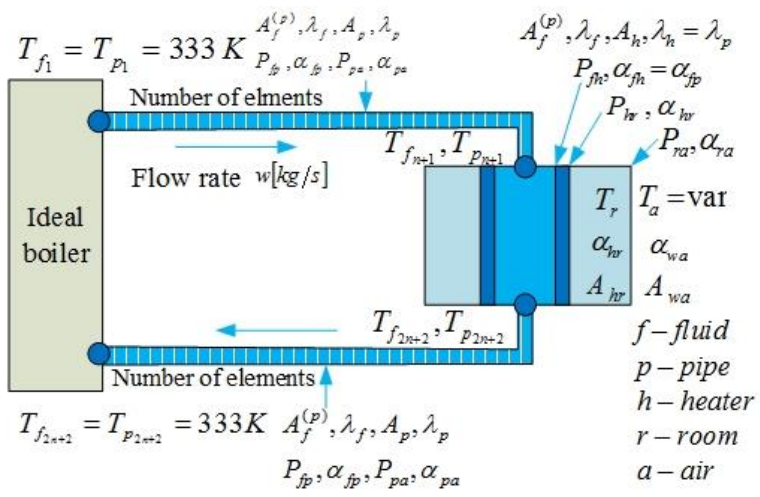

b

Fig. 1 The scheme of the mathematical model of the heat transfer stand (a) and the model parameters used in computations (b)

\section{Finite element model}

The structural vector of nodal temperatures of the model is presented in Fig. 1, a. The supply and return pipes each may be discretized into $\mathrm{n} F \mathrm{FE}$. The heater is presented as a single FE between nodes $n+1$ and $n+2$. Each node of the model is characterized by two temperatures (fluid and pipe). The full structural nodal temperatures vector is ob- 
tained by supplementing the nodal temperatures vector of the pipes and heater by the temperature $T_{r}$ of the HS. The full length of the nodal temperatures vector is $2 n+3$. It should be noted that the latter node presenting the HS is described by only one temperature value, whereas all the remaining nodes are characterized by two temperatures (fluid and pipe).

The full model of the system is composed of the following types of FE.

Diffusive (conductive) heat exchange in the pipe wall:

$$
\begin{gathered}
c_{p p}\left[\begin{array}{cc}
1 & 0 \\
0 & 1
\end{array}\right]\left\{\begin{array}{c}
\dot{T}_{p_{t}} \\
\dot{T}_{p_{t+1}}
\end{array}\right\}+\left(k_{p p}\left[\begin{array}{cc}
1 & -1 \\
-1 & 1
\end{array}\right]+k_{p a}\left[\begin{array}{cc}
1 & 0 \\
0 & 1
\end{array}\right]\right) \times \\
\times\left\{\begin{array}{c}
T_{p_{t}} \\
T_{p_{t+1}}
\end{array}\right\}=k_{p a} T_{a}\left\{\begin{array}{l}
1 \\
1
\end{array}\right\}+\left\{\begin{array}{l}
Q_{p 1} \\
Q_{p 2}
\end{array}\right\},
\end{gathered}
$$

where $k_{p p}=\frac{\lambda_{p} A_{p}}{L_{p}} ; k_{p a}=\frac{\alpha_{p a} P_{p a} L_{p}}{2} ; c_{p p}=\frac{c_{p} \rho_{p} A_{p} L_{p}}{2}$ the combined physical meaning of the last term at the left-hand side and of first term at the right hand side of Eq. (1) is the power of the convective heat exchange between the external surface of the pipe and the air. The FE is used inbetween of the degrees of freedom (DOF) corresponding to the fluid and pipe temperatures of all nodes of the supply and return pipes.

Diffusive (conductive) heat exchange in the fluid within the pipe FE and heat transfer due to the fluid flow rate $w$ :

$$
\begin{aligned}
& c_{f f}^{(p)}\left[\begin{array}{ll}
1 & 0 \\
0 & 1
\end{array}\right]\left\{\begin{array}{c}
\dot{T}_{f_{i}} \\
\dot{T}_{f_{i+1}}
\end{array}\right\}+\left(k_{f f}^{(p)}\left[\begin{array}{cc}
1 & -1 \\
-1 & 1
\end{array}\right]+w c_{f}\left[\begin{array}{cc}
0 & 0 \\
-1 & 1
\end{array}\right]\right) \times \\
& \times\left\{\begin{array}{c}
T_{f_{i}} \\
T_{f_{i+1}}
\end{array}\right\}=\left\{\begin{array}{l}
Q_{f 1}^{(p)} \\
Q_{f 2}^{(p)}
\end{array}\right\}, k_{f f}^{(p)}=\frac{\lambda_{f} A_{f}^{(p)}}{L_{p}} ; c_{f f}^{(p)}=\frac{c_{f} \rho_{f} A_{f}^{(p)} L_{p}}{2},
\end{aligned}
$$

where the physical meaning of the last term at the left-hand side of Eq. (2) is the heat transfer due to the fluid flow rate. The FE is used in-between the DOF corresponding to fluid temperatures of all adjacent nodes of the pipes.

Convective heat exchange between the fluid and pipe:

$$
\frac{\alpha_{p f} P_{f p} L_{p}}{2}\left[\begin{array}{cc}
1 & -1 \\
-1 & 1
\end{array}\right]\left\{\begin{array}{l}
T_{f_{i}} \\
T_{p_{i}}
\end{array}\right\}=\left\{\begin{array}{l}
Q_{p f 1} \\
Q_{p f 2}
\end{array}\right\},
$$

where $k_{p f}=\frac{\alpha_{p f} P_{f p} L_{p}}{2}$.

The FE is used in-between the DOF corresponding to the fluid and pipe temperatures of all nodes of supply and return pipes. No heat capacity terms are employed in this FE element equation as the heat exchange takes place through the hypothetical separating surface between the fluid and adjacent pipe wall.

Diffusive heat exchange in the heater wall:

$c_{h h}\left[\begin{array}{cc}1 & 0 \\ 0 & 1\end{array}\right]\left\{\begin{array}{l}\dot{T}_{f_{n+1}} \\ \dot{T}_{f_{n+2}}\end{array}\right\}+\frac{\lambda_{h} A_{h}}{L_{p}}\left[\begin{array}{cc}1 & -1 \\ -1 & 1\end{array}\right]\left\{\begin{array}{c}T_{p_{n+1}} \\ T_{p_{n+2}}\end{array}\right\}=\left\{\begin{array}{l}Q_{h 1} \\ Q_{h 2}\end{array}\right\}$, where $k_{h h}=\frac{\lambda_{h} A_{h}}{L_{p}} ; c_{h h}=\frac{c_{h} \rho_{h} A_{h} L_{h}}{2}$

The element is used in-between the DOF corresponding to the pipe temperatures of heater nodes $n+1$ and $n+2$.

Diffusive (conductive) heat exchange in the fluid within the heater FE and heat transfer due to the fluid flow rate $w$ :

$$
\begin{aligned}
& c_{f f}^{(h)}\left[\begin{array}{ll}
1 & 0 \\
0 & 1
\end{array}\right]\left\{\begin{array}{c}
\dot{T}_{f_{n+1}} \\
\dot{T}_{f_{n+2}}
\end{array}\right\}+\left(k_{f f}^{(h)}\left[\begin{array}{cc}
1 & -1 \\
-1 & 1
\end{array}\right]+w c_{f}\left[\begin{array}{cc}
0 & 0 \\
-1 & 1
\end{array}\right]\right) \times \\
& \times\left\{\begin{array}{c}
T_{f_{n+1}} \\
T_{f_{n+2}}
\end{array}\right\}=\left\{\begin{array}{l}
Q_{f 1}^{(h)} \\
Q_{f 2}^{(h)}
\end{array}\right\}, k_{f f}^{(h)}=\frac{\lambda_{f} A_{f}^{(h)}}{L_{h}} ; c_{f f}^{(h)}=\frac{c_{f} \rho_{f} A_{f}^{(h)} L_{h}}{2}
\end{aligned}
$$

Convective heat exchange between the fluid and the heater:

$$
k_{f h}\left[\begin{array}{cc}
1 & -1 \\
-1 & 1
\end{array}\right]\left\{\begin{array}{c}
T_{f_{i}} \\
T_{p_{i}}
\end{array}\right\}=\left\{\begin{array}{l}
Q_{f h 1} \\
Q_{f h 2}
\end{array}\right\}, k_{f h}=\frac{\alpha_{f h} P_{f h} L_{h}}{2} .
$$

The element is used in-between the DOF corresponding to the fluid and the heater wall temperatures of nodes $n+1$ and $n+2$ (see explanation of formula (3) regarding the absence of the heat capacity terms).

Convective heat exchange between the heater and HS:

$$
\frac{c_{a} \rho_{a} V_{r}}{2}\left[\begin{array}{cc}
0 & 0 \\
0 & 1
\end{array}\right]\left\{\begin{array}{c}
\dot{T}_{h_{i}} \\
\dot{T_{r}}
\end{array}\right\}+k_{h r}\left[\begin{array}{cc}
1 & -1 \\
-1 & 1
\end{array}\right]\left\{\begin{array}{c}
T_{h_{i}} \\
T_{r}
\end{array}\right\}=\left\{\begin{array}{c}
Q_{h r} \\
Q_{r}
\end{array}\right\}
$$

where $k_{h r}=\frac{\alpha_{h r} P_{h r} L_{h}}{2} ; c_{r r}=\frac{c_{a} \rho_{a} V_{r}}{2}$.

Two identical FE are used in-between the heater wall temperature DOF of nodes $n+1$ and $n+2$ of the heater, on one side, and the DOF corresponding to the HS temperature of node $2 n+3$, on the other side.

Convective heat exchange between the HS and outer air:

$$
\frac{C_{w}}{2}\left[\begin{array}{cc}
1 & 0 \\
0 & 1
\end{array}\right]\left\{\begin{array}{c}
\dot{T}_{r} \\
\dot{T}_{a}
\end{array}\right\}+k_{r a}\left[\begin{array}{cc}
1 & -1 \\
-1 & 1
\end{array}\right]\left\{\begin{array}{c}
T_{r} \\
T_{a}
\end{array}\right\}=\left\{\begin{array}{l}
Q_{r} \\
Q_{a}
\end{array}\right\},
$$

where $k_{r a}=\alpha_{r a} A_{r a}$.

The element is used in-between the DOF of HS air temperature and the DOF of outer air temperature. In addition the heat capacity of the external wall of the HS is considered as $C_{w}$.

In case the supply and return pipes are presented each by a single FE, the structural equation obtained by assembling Eqs. (1)-(8) reads as (9).

$$
\boldsymbol{C} \dot{\boldsymbol{T}}+\left(\boldsymbol{K}+w \boldsymbol{K}_{w}\right) \boldsymbol{T}=\boldsymbol{S} T_{a} .
$$

Assume the temperatures of the fluid and pipes at the boiler wall $\boldsymbol{T}_{b}=\left\{T_{\text {fin }}, T_{\text {pin }}, T_{\text {fout }}, T_{\text {pout }}\right\}^{T}$ are known. The mask IS is used, where corresponding DOF are marked by „ones" as: 


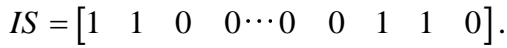

Eq. (9) is re-arranged by employing the matrix blocs corresponding to "ones" and "zeros" in the mask as:

$$
\boldsymbol{C}^{00} \dot{\boldsymbol{T}}^{0}+\left(\boldsymbol{K}^{00}+w(t) \boldsymbol{K}_{w}^{00}\right) \boldsymbol{T}=\boldsymbol{S}^{0} T_{a}+\boldsymbol{S}_{b}(t),
$$

where $\boldsymbol{S}_{b}=-\left(\boldsymbol{K}^{01}+w(t) \boldsymbol{K}_{w}^{01}\right) \boldsymbol{T}_{b}$.

\section{Computational example}

In the initial state the system is connected to the boiler and flow rate equals zero. The thermal equilibrium condition is presented in Fig. 2.

The flow rate of heating fluid $0.1 \mathrm{~kg} / \mathrm{s}$ supplied from time moment $500 \mathrm{~s}$ until $10000 \mathrm{~s}$. Fig. 3 presents the distribution of temperatures in the system at time moments $5200 \mathrm{~s}, 10080 \mathrm{~s}, 17040 \mathrm{~s}$. Fig. 4 presents variation in time of heater inlet, heater outlet and HS temperatures.

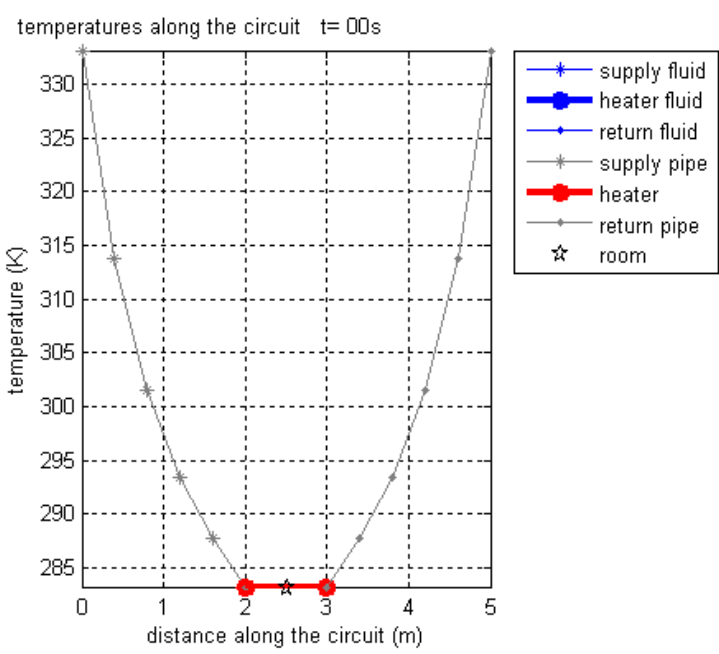

Fig. 2 Initial temperatures along the heating circuit (zero flow rate)

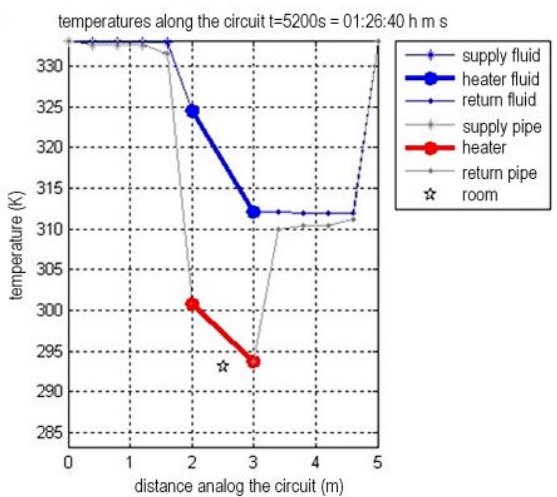

a

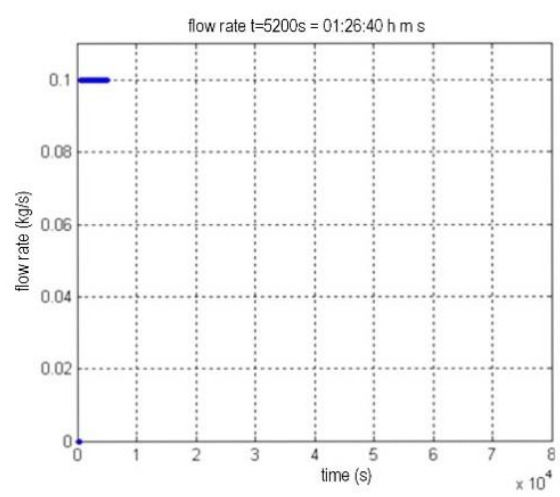

b

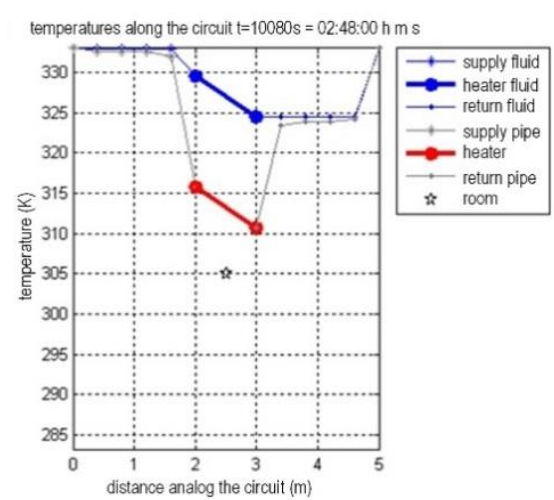

c

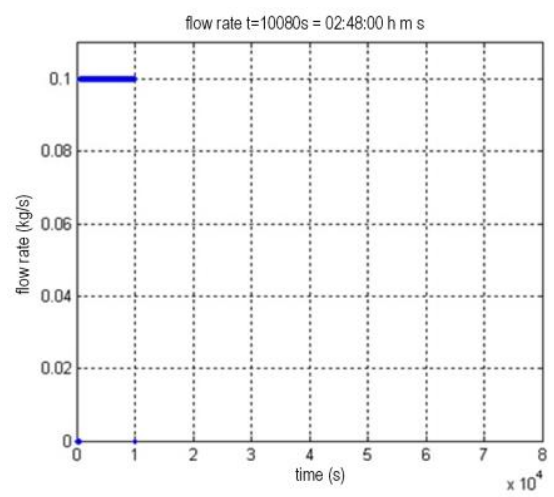

d

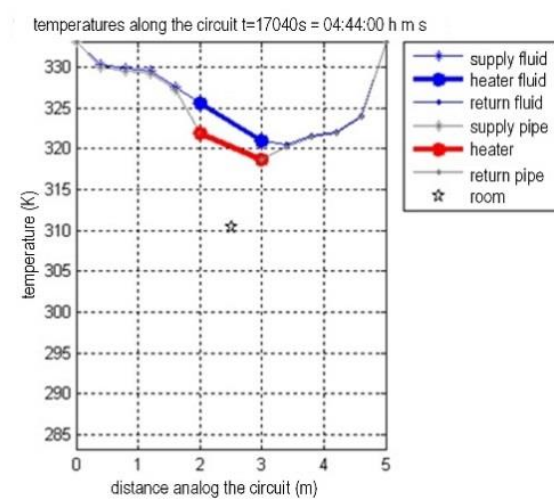

$\mathrm{e}$

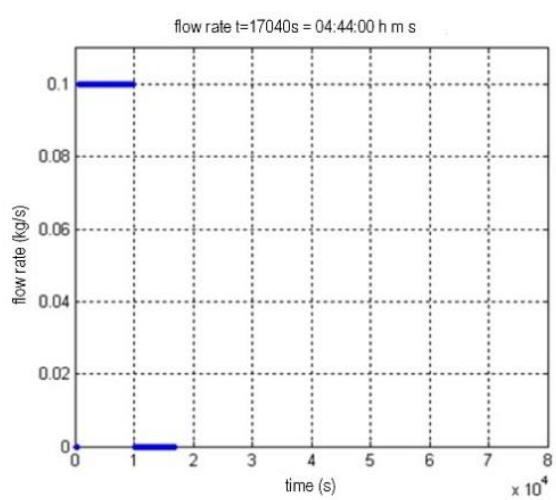

f

Fig. 3 Distribution of temperatures in the heating circuit (a, c, e) and flow rates (b, d, f) at time moments $5200 \mathrm{~s}$ (a, b), $10080 \mathrm{~s}(\mathrm{c}, \mathrm{d}), 17040 \mathrm{~s}(\mathrm{e}, \mathrm{f})$

\section{Minimization of the flow rate for maintaining the prescribed temperature of the heated space}

It is necessary to find the control law ensuring the minimum power consumption by the pump in order to maintain the prescribed temperature value in the HS. Generally, the control law of the heating fluid flow rate can be obtained by solving the optimal control problem as fixed flow rate $\tilde{w}$, start-stop control: $\min _{\tilde{\tau}, \tau} \frac{\tilde{\tau}}{\tau}$.

$$
\left\{\begin{aligned}
\boldsymbol{C}^{00} \dot{\boldsymbol{T}}^{0} & +\left(\boldsymbol{K}^{00}+w(t) \boldsymbol{K}_{w}^{00}\right) \boldsymbol{T}^{0}=\boldsymbol{S}^{0} T_{a}- \\
& -\left(\boldsymbol{K}^{01}+w(t) \boldsymbol{K}^{01}+w(t) \boldsymbol{K}_{w}^{01}\right) \boldsymbol{T}_{b} ; \\
\boldsymbol{T}^{0}(0)= & \boldsymbol{T}^{0}(\tau) ; \\
w(t)= & \begin{cases}\tilde{w}, & 0<t \leq \tilde{\tau} ; \\
0, & \tilde{\tau}<t \leq \tau,\end{cases}
\end{aligned}\right.
$$

where $\tau$ is period of the rate pulse; $\tilde{\tau}$ is rate pulse duration during the period. 


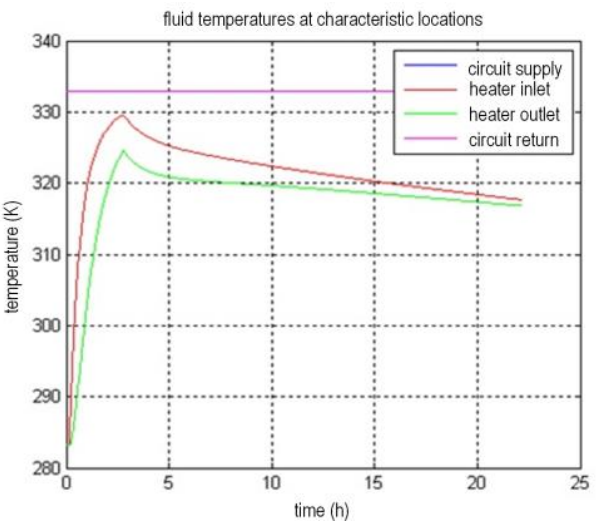

a

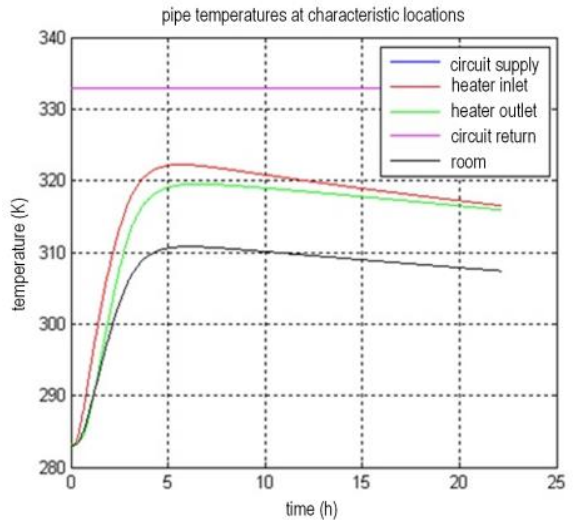

b

Fig. 4 Variation in time of the fluid temperatures (a) and pipe temperatures (b) at heater inlet, at heater outlet and in the HS, where heating fluid flow rate $0.1 \mathrm{~kg} / \mathrm{s}$ is supplied from $500 \mathrm{~s}$ until $10000 \mathrm{~s}$

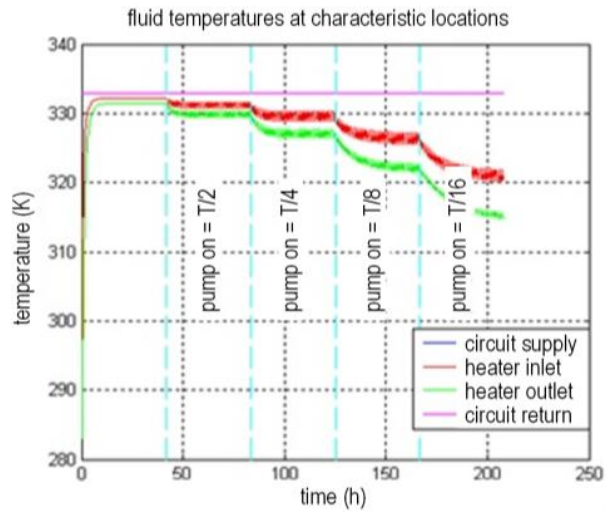

$\mathrm{a}$

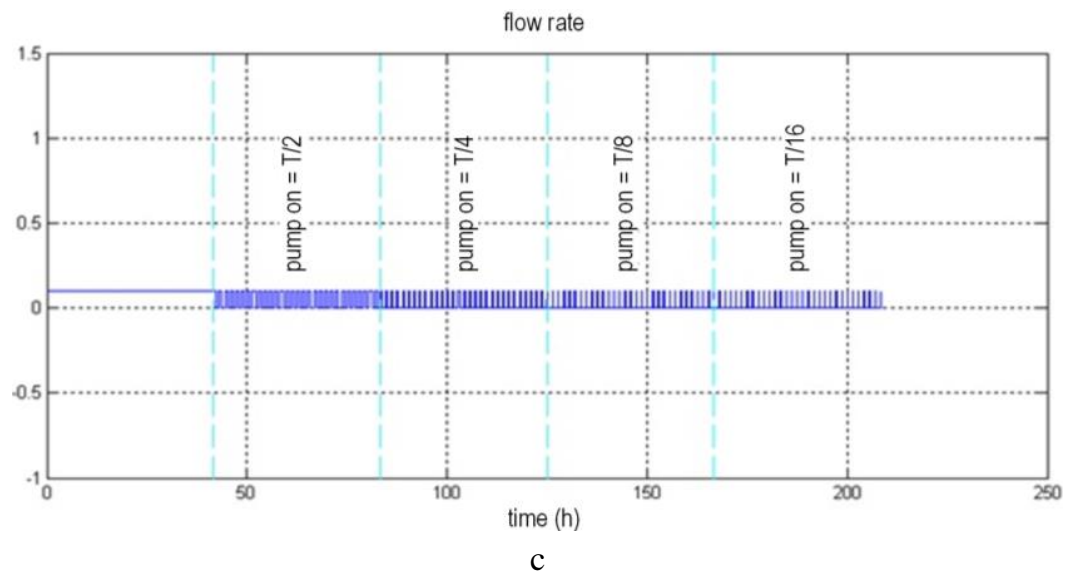

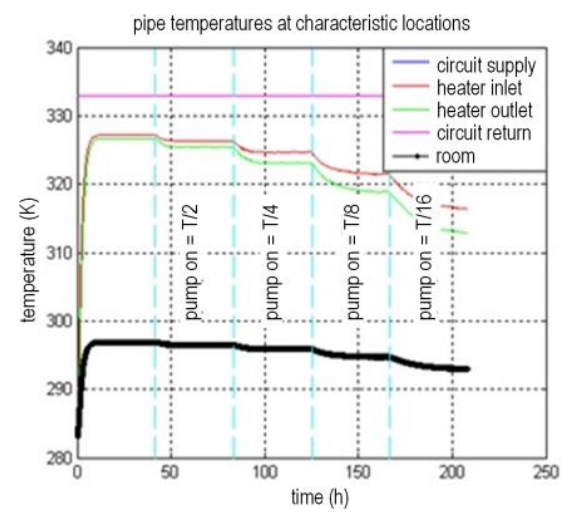

b

Fig. 5 Variation of the fluid temperatures (a) and pipe temperatures (b) in time in case of five heating stages (c). During each stage the individual <pump on> time parts $1,1 / 2,1 / 4,1 / 8,1 / 16$ during the pulse time period are employed. The pulse period is $1 \mathrm{~h} 23 \mathrm{~min} 20 \mathrm{~s}$ (5000 s)

Fig. 5 presents the time relationships of the fivestage process:

- During the 1 st heating stage a constant heating fluid rate $\tilde{w}$ is maintained in the circuit until steady HS temperature is reached.

- During the 2nd heating stage the heating fluid rate is controlled as periodic pulses, where <pump on> time part comprises one-half of the pulse time period $\frac{\tilde{\tau}}{\tau}=\frac{1}{2}$. The drop of the average temperature in the HS is only $0.3^{\circ} \mathrm{C}$ compared with the steady value of the temperature at continuous heating. The temperature drop of the return fluid is about $1.5^{\circ} \mathrm{C}$. 


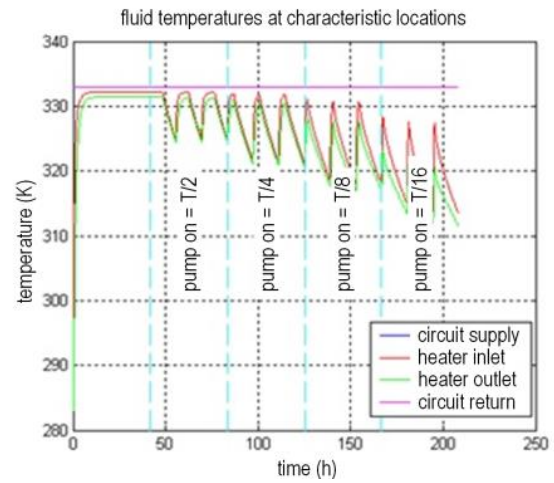

a

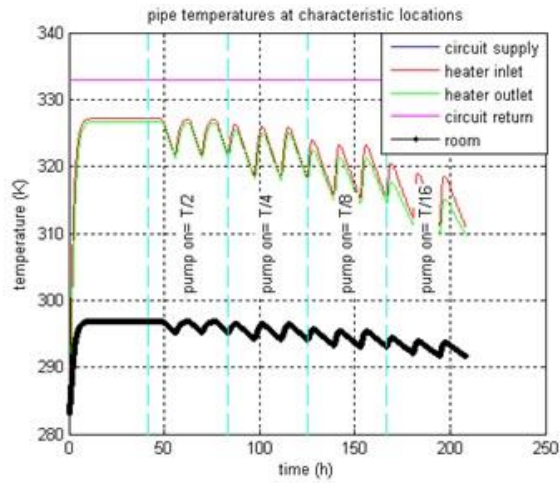

b

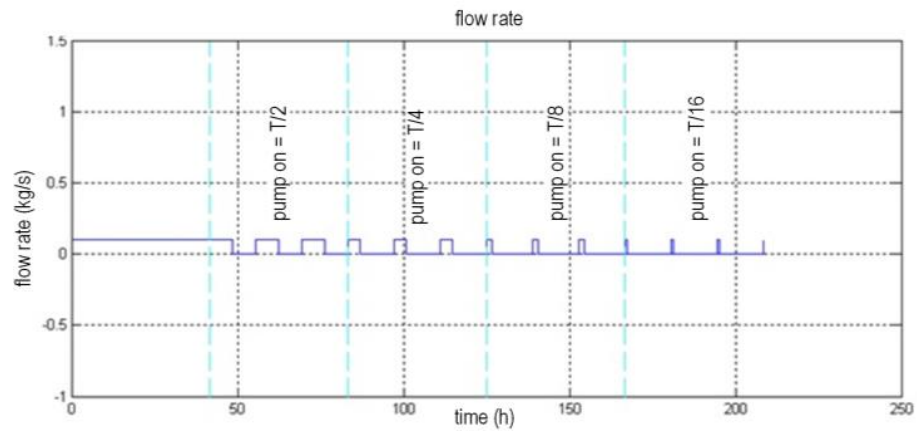

$\mathrm{c}$

Fig. 6 Variation of the fluid temperatures (a) and pipe temperatures (b) in time in case of five heating stages (c). During each stage the individual <pump on> time parts $1,1 / 2,1 / 4,1 / 8,1 / 16$ during the pulse time period are employed. The pulse period is $1 \mathrm{~h} 23 \mathrm{~min} 20 \mathrm{~s}(5000 \mathrm{~s})$

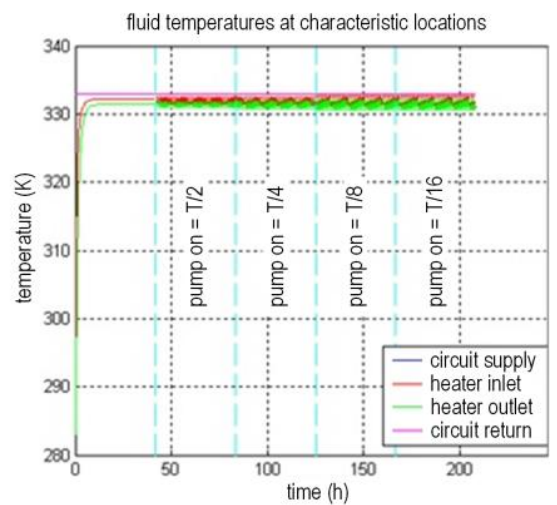

a

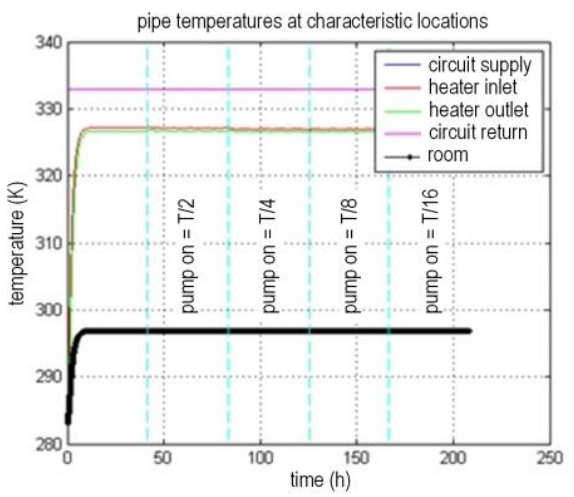

$\mathrm{b}$

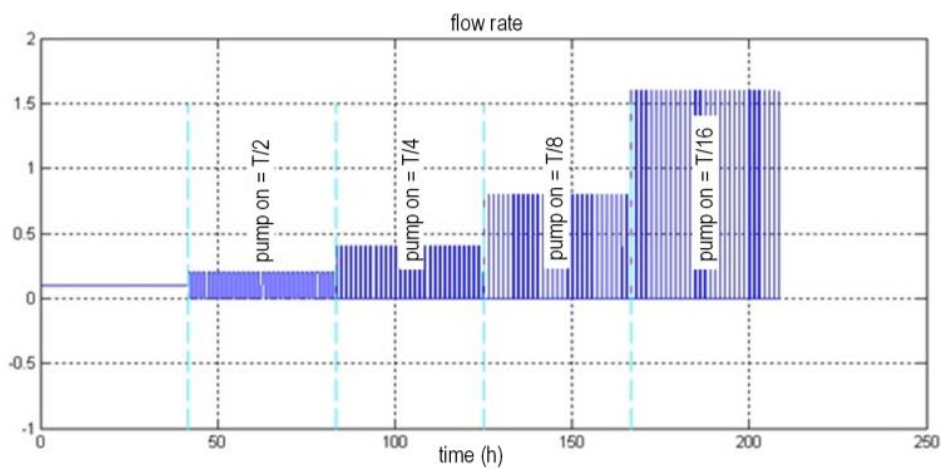

$\mathrm{c}$

Fig. 7 Variation of the fluid temperatures (a) and pipe temperatures (b) in time in case of five heating stages (c). During each stage the constant average flow rate is maintained. During each stage the individual <pump on> time parts 1 , $1 / 2,1 / 4,1 / 8,1 / 16$ during the pulse period are employed, and the <pump on> flow rate is multiplied by $1,2,4,8$ and 16 correspondingly. The pulse period is $1 \mathrm{~h} 23 \mathrm{~min} 20 \mathrm{~s}(5000 \mathrm{~s})$

Fig. 6 demonstrates that the duration of the activity time of the pump during the pulse time period only mi- nutely influences the value of average temperature in the HS, though temperature fluctuations with respect to aver- 
age values become larger as the activity time of the pump decreases.

In case constant average flow rate is maintained during each heating stage, the average temperature of the HS remains constant, Fig. 7.

The relationships of the steady temperatures in the heated space against the <pump off> time part during the period at different temperatures of the boiler are presented in Fig. 8. The curves demonstrate that the decrease of temperatures of the HS due to the decrease of the average flow rate is almost independent on the temperature of the boiler, until the <pump on> time part during the pulse time period is not less than $1 / 4$.

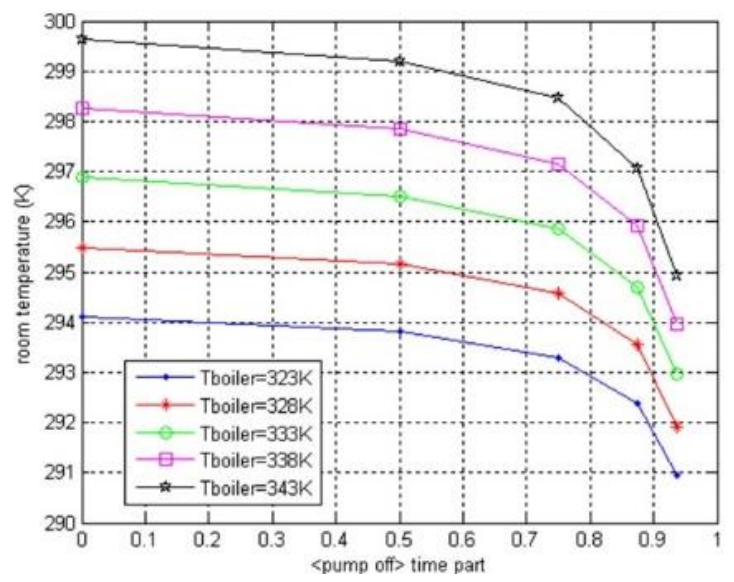

Fig. 8 Dependencies of average temperatures in the heated space against the <pump off > time part during the period at different boiler temperature values

\section{Conclusions}

1. The circulation pump activation law in the form of periodic pulses enables to save the average electric power necessary for maintaining the prescribed average temperature of the heated space. As the average electric power was halved, the temperature drop in the heated space decreased by only $\sim 0.3^{\circ} \mathrm{C}$;

2. In case the average fluid flow rate provided by the pump is maintained constant, the variation of the pulse duration does not influence the average temperature value in the heated space;

3. Until the <pump on> time part comprises not less than $1 / 4$ of the period, the drop of the temperature of the heated space is less than $1^{\circ} \mathrm{C}$.

\section{Acknowledgement}

This research was funded by EU Structural Funds project "In-Smart" (Nr. VP1-3.1-ŠMM-10-V-02-012), Ministry of Education and Science, Lithuania.

\section{References}

1. Pascal Bruegger; Vincenzo Pallotta; Beat Hirsbrunner 2007. Smart Heating System: Optimizing house heating systems by integrating user motion, Technical Report 07-12, University of Fribourg, Switzerland.

2. Pallotta Vincenzo; Bruegger Pascal; Hirsbrunner, Béat. 2008. Smart Heating Systems: optimizing heating systems by kinetic-awareness, in: Proceedings of 3rd ICDIM conference, IEEE Press, IEEE, London.

3. Hana, H.J.; Jeona, Y.I.; Limb, S.H.; Kimc, W.W.;
Chend, K. 2010. New developments in illumination, heating and cooling technologies for energy-efficient buildings, Energy 35(6): 2647-2653.

http://dx.doi.org/10.1016/j.energy.2009.05.020.

4. Marie Munster; Peter Meibom 2011. Optimization of use of waste in the future energy system, Energy 36(3): 1612-1622.

http://dx.doi.org/10.1016/j.energy.2010.12.070.

5. Arif Hepbasli; Yildiz Kalinc 2009. A review of heat pump water heating systems, Renewable and Sustainable Energy Reviews 13: 1211-1229.

http://dx.doi.org/10.1016/j.rser.2008.08.002.

6. Samuel Idowu; Christer Ahlund; Olov Schelen 2014. Machine learning in district heating system energy optimization, 2014 IEEE International Conference on Pervasive Computing and Communications Work in Progress.

R. Barauskas, V. Grigaliunas, M. Gudauskis, L. Obcarskas, K. Sarkauskas, A. Vilkauskas, A. Zvironas

\section{COMPUTATIONAL MODEL OF THE HEATING SYSTEM}

S u m m a r y

The primary objective of smart heating systems is to maintain a comfortable temperature in the rooms.

The aim of our paper is to propose a method for increasing electric efficiency of older houses heating systems by making simple changes in heating pumps. It has been demonstrated that appropriate start-stop control of the pump may result in significant reduction of the overall pump operation time.

The boiler is assumed to be an ideal one, the temperatures of which are always constant and equal $\left(60^{\circ} \mathrm{C}\right)$ at the zones of connection of the supply and return pipes. In this model the temperature of the heated space (HS) is assumed to be uniform throughout its volume. The temperature of the HS is influenced by the heat exchange processes through its outer surface, as well as, through the surface of the heater in contact with HS. The heat conductivity and surface convection coefficients of the heater are assessed by assuming it as an equivalent pipe. The transient heat transfer problem is solved under assumption that the heating fluid flow rate is a known time function. The aim of the analysis is to find the time law of the fluid flow rate, which ensures minimum power consumption by the pump. Steady temperature of HS within given tolerances must be maintained.

The relationships of the steady temperatures in the heated space against the <pump off> time part during the period at different temperatures of the boiler are presented in diagram. The curves demonstrate that the decrease of temperatures of the HS due to the decrease of the average flow rate is almost independent on the temperature of the boiler, until the <pump on> time part during the pulse time period is not less than $1 / 4$.

Keywords: heating pump, fluid flow rate, convective heat transfer.

Received May 12, 2015

Accepted January 06, 2016 